\title{
O GERENCIAMENTO DA PANDEMIA DA GRIPE A (H1N1): UMA VISÃo ALTERNATIVA
}

\section{Germán Velásquez*}

\section{RESUMO}

Este artigo tem por objetivo analisar os interesses comerciais não relacionados à saúde pública que surgiram durante a condução da pandemia da gripe A (H1N1) no Hemisfério Norte. Também busca refletir como a preparação para esta pandemia poderia se tornar um peso para os países do Hemisfério Sul. Além disso, este trabalho examina a complexidade da divulgação científica pelos meios de comunicação em casos de surtos epidêmicos graves.

\section{Palavras-chave:}

Meios de Comunicação; Organização Mundial da Saúde; Pandemia H1N1.

\section{ABSTRACT}

The purpose of this paper is to analyse the commercial non-sanitary interests of the management of the pandemic influenza preparedness in the Global North and how this preparedness for the pandemic could be an additional burden for the countries of the Global South. Moreover, this paper examines the complexity of scientific outreach to society through the media in the case of serious epidemic outbreaks.

(*) Doutor, Universidade Sorbonne (Paris). Conselheiro especial sobre Saúde e Desenvolvimento do Centro Sul (Genebra). Genebra - Suiça. E-mail: south@southcentre.org.

Texto recebido em 16.07.2012. Revisado em 09.04.2012. Aprovado em 22.10.2012. 


\section{Keywords:}

H1N1 Pandemic; World Health Organization; Media.

\section{INTRODUÇÃO}

Jornalistas são frequentemente criticados e tachados de irresponsáveis, exagerados e sensacionalistas quando cobrem desastres naturais ou surtos epidêmicos graves. Porém, uma reflexão sobre a comunicação da pandemia da gripe $\mathrm{A}(\mathrm{H} 1 \mathrm{~N} 1)^{(1)}$ mostra que houve um maior entendimento entre os meios de comunicação do que entre os chamados "cientistas" das organizações internacionais e ministérios da Saúde dos países desenvolvidos. A partir de junho-julho de 2009, a imprensa começou a questionar os cientistas e as autoridades públicas que não se mostraram capazes de responder às perguntas levantadas. Hoje, três anos depois, os jornalistas podem ter acertado em formular aquelas questões e inquietações que conduziram avaliações externas independentes, como a do Conselho Europeu ${ }^{(2)}$ e a do Parlamento Francês. ${ }^{(3)}$

Antes de tudo, é importante lembrar que um terço da população mundial não tem acesso regular a medicamentos e que milhares de pessoas morrem diariamente em razão dessa situação(4). Segundo o Programa Conjunto das Nações Unidas sobre HIV/Aids (Unaids), 20 milhões de pessoas nos países em desenvolvimento deveriam estar recebendo tratamento antirretroviral, mas apenas 5,2 milhões ${ }^{(5)}$ recebem efetivamente essas drogas. Isso significa que muitos dos 15 milhões de pacientes sem acesso aos medicamentos podem morrer nos próximos dois a quatro anos, se não houver uma mudança radical.

(1) Segundo a OMS um alerta de pandemia na fase 5 carateriza-se pela contaminação de homem para homem em pelo menos dois países. A fase 6 (o estágio mais avançado) de uma pandemia tem como característica surtos em níveis comunitários em pelo menos um outro país em uma região diferente. WORLD HEALTH ORGANIZATION. Current WHO phase of pandemic alert for Pandemic (H1N1) 2009. Disponível em: <http://www.who.int/csr/disease/swineflu/phase/en>. Acesso em: 4 set. 2012. A pandemia $\mathrm{H} 1 \mathrm{~N} 1$ foi descrita pela OMS, pela primeira vez, em abril de 2009; em 10 de agosto de 2010, a diretora geral da organização, Margareth Chan, anunciou o fim da pandemia H1N1.

(2) CASSEL, Ingri. H1N1 pandemic scam cost \$billions worldwide. European Parliamentary Assembly holds public hearing to investigate "what went wrong". Idaho Observer, Strasbourg, France. Feb. 10th, 2010. Disponível em: <http://www.proliberty.com/observer/20100234.html>. Acesso em: 26 jul. 2012. (3) ASSEMBLÉE NATIONALE. Rapport. Au nom de la Commission d'enquête sur la manière dont a été programmée, expliquée et gérée la campagne de vaccination contre la grippe $A(H 1 N 1)$. Disponível em: $<$ http://www.assemblee-nationale.fr/13/rap-enq/r2698.asp>. Acesso em: 26 jul. 2012.

(4) De acordo com a OMS, "Cerca de nove milhões de crianças menores de cinco anos morrem todos os dias, muitas delas em situações que poderiam ser tratadas com medicamentos eficazes e seguros". WORLD HEALTH ORGANIZATION. Medicines: medicines for children. Fact Sheet n. 341, June 2010. Disponível em: <http://www.who.int/mediacentre/factsheets/fs341/en/index.html>. Acesso em: 26 jul. 2012.

(5) UNAIDS. Global Report 2010. Disponível em: <http://www.unaids.org/globalreport/Global_report. htm>. Acesso em: 26 jul. 2012. 


\section{O ESTOQUE DE OSELTAMIVIR ${ }^{(6)}$}

No caso da gripe aviária (H5N1), desde 2005, existem estoques da droga Oseltamivir (Tabela 1), ${ }^{(7)}$ cuja eficácia não foi comprovada e que é destinada a uma doença que ainda não emergiu (uma situação nunca vista na história da medicina). São estoques que podem atender de $20 \%$ a $50 \%$ da população em muitos países desenvolvidos. No entanto, eles terão que ser destruídos porque os medicamentos perderão a validade nos próximos anos.

Os eventos iniciaram em 2005 com a ameaça da gripe aviária causada pelo vírus H5N1. Se olharmos a história da saúde pública através do século 20, veremos que têm existido centenas de variações de vírus gripais. No entanto, fora a gripe $A(\mathrm{H} 1 \mathrm{~N} 1)$, que é de origem suína, quantos deles atualmente infectam humanos? A resposta: somente quatro ${ }^{(8)}$.

No caso da gripe aviária (H5N1), os cientistas afirmaram, para os meios de comunicação, que ela era altamente patogênica. Em agosto de 2005, o chefe de gabinete do escritório da diretora geral da Organização Mundial da Saúde (OMS) anunciou, por meio de press release, que 150 milhões de pessoas no mundo poderiam morrer em razão de uma possível pandemia. Em outubro do mesmo ano, somente dois meses depois, a OMS disse que o número poderia ser de 20 milhões de pessoas. Em janeiro de 2006, anunciou-se que seria de cerca de dois a 7,4 milhões. Em outubro de 2010, a OMS informou que ocorreram 331 mortes $^{(9)}$ (passados nove anos do primeiro óbito), com maior concentração de casos na Indonésia e no Vietnã ${ }^{(10)}$. Doenças mais comuns do que a gripe aviária apresentam uma taxa de mortalidade maior.

Essa disparidade entre os resultados esperados e aqueles observados provoca um questionamento sobre como os números de óbitos são calculados. Quando a OMS previu que entre dois e 7,4 milhões de pessoas poderiam morrer, a resposta que epidemiologistas apresentaram foi a de que esse cálculo foi feito com base na experiência histórica. De acordo com os registros históricos,

(6) Oseltamivir é uma droga antiviral, comercializada com o nome de Tamiflu.

(7) WORLD HEALTH ORGANIZATION. Donation of three million treatments of oseltamivir to WHO will help early response to an emerging influenza pandemic. Aug. 24th 2005. Disponível em: <http:// www.who.int/mediacentre/news/releases/2005/pr36/en/>. Acesso em: 27 jul. 2012.

(8) CENTER FOR DISEASE CONTROL AND PREVENTION. Transmission of Influenza A Viruses Between Animals and People. Subtypes that have caused widespread illness in people either in the past or currently are H3N2, H2N2, H1N1, and H1N2. Disponível em: <http://www.cdc.gov/flu/avian/ gen-info/transmission.htm>. Acesso em: 11 nov. 2012.

(9) WORLD HEALTH ORGANIZATION/GIP. Cumulative number of confirmed human cases of avian influenza H5N1 reported to WHO, 2003-2011. Aug. 19th 2011. Disponível em: <http://www.who. int/influenza/human_animal_interface/EN_GIP_20110819CumulativeNumberH5N1casesN.pdf>. Acesso em: 27 jul. 2012.

(10) A Indonésia relatou 146 casos e o Vietnã, 59. WORLD HEALTH ORGANIZATION/GIP. Cumulative number of confirmed human cases of avian influenza H5N1 reported to WHO, 2003-2011, cit. 
Tabela 1 - Comparação de estoques internacionais de Oseltamivir/Zanamivir

\begin{tabular}{|c|c|c|c|}
\hline $\begin{array}{l}\text { País } \\
\text { (população } \\
\text { em milhão) }\end{array}$ & $\begin{array}{c}\text { Tamanho e composição } \\
\text { do estoque }\end{array}$ & Usos & Observações \\
\hline $\begin{array}{l}\text { Canadá } \\
\text { (33) }\end{array}$ & $\begin{array}{c}\sim 25 \% \text { da população } \\
\text { NAS: } \\
\text { 50,7 milhões de doses de } \\
\text { Oseltamivir } \\
5 \text { milhões de doses de } \\
\text { Zanamivir } \\
\text { Mais outros estoques } \\
\text { federais e particulares } \\
\text { ( 80 milhões no total) }\end{array}$ & $\begin{array}{l}\text { Tratamento } \\
\text { inicial; contenção } \\
\text { rápida; controle } \\
\text { de surtos; } \\
\text { profilaxia pós- } \\
\text { exposição na } \\
\text { fase } 4 / 5\end{array}$ & $\begin{array}{c}\text { Estoques } \\
\text { particulares podem } \\
\text { ser apresentados } \\
\text { para profilaxia pré- } \\
\text { exposição }\end{array}$ \\
\hline $\begin{array}{l}\text { Estados } \\
\text { Unidos } \\
\text { (304) }\end{array}$ & $\begin{array}{c}25 \% \text { da população } \\
\text { (meta 35\%) } \\
\text { Quase todo o estoque é } \\
\text { de Oseltamivir }\end{array}$ & $\begin{array}{l}\text { Tratamento } \\
\text { inicial; contenção } \\
\text { rápida (profilaxia } \\
\text { a ser coberta } \\
\text { pelo estoque } \\
\text { privado) }\end{array}$ & $\begin{array}{l}\text { Apoio a estoque } \\
\text { particular; tem } \\
\text { um programa de } \\
\text { extensão da vida útil } \\
\text { bem estabelecido }\end{array}$ \\
\hline $\begin{array}{l}\text { Reino } \\
\text { Unido } \\
(61)\end{array}$ & $\begin{array}{c}\text { 25\% da população; } \\
\text { compromisso recente } \\
\text { para aumentar para } \\
50 \% \text { da população } \\
\text { (32,8 milhões) } \\
2 / 3 \text { de Oseltamivir } \\
1 / 3 \text { de Zanamivir }\end{array}$ & $\begin{array}{l}\text { Tratamento } \\
\text { inicial - } \\
\text { suficiente para } \\
\text { uma pandemia } \\
\text { severa e pouca } \\
\text { quantidade para } \\
\text { profilaxia pré- } \\
\text { exposição }\end{array}$ & $\begin{array}{l}\text { Profilaxia pós- } \\
\text { exposição em } \\
\text { domicílios em } \\
\text { consideração; busca } \\
\text { alternativas para o } \\
\text { estoque prestes a } \\
\text { expirar }\end{array}$ \\
\hline $\begin{array}{l}\text { França } \\
(62)\end{array}$ & $\begin{array}{c}50 \% \text { da população (pode } \\
\text { dividir com outros países } \\
\text { da União Europeia) } \\
240 \text { milhões de doses de } \\
\text { Oseltamivir } \\
90 \text { milhões de doses de } \\
\text { Zanamivir }\end{array}$ & $\begin{array}{c}\text { Tratamento } \\
\text { inicial; profilaxia } \\
\text { pré-exposição na } \\
\text { fase } 4 / 5\end{array}$ & $\begin{array}{l}\text { Estoque começou } \\
\text { a expirar em } 2008 \text {; } \\
\text { busca por uma } \\
\text { extensão da vida útil }\end{array}$ \\
\hline $\begin{array}{l}\text { Japão } \\
\text { (127) }\end{array}$ & $\begin{array}{l}\text { 23\% da população; } \\
\text { objetivo é } 45 \% \text { (podem } \\
\text { compartilhar com a } \\
\text { Tailândia e outros } \\
\text { países asiáticos) } \\
240 \text { milhões de doses } \\
\text { de Oseltamivir } \\
90 \text { milhões de } \\
\text { doses de Zanamivir }\end{array}$ & $\begin{array}{l}\text { Tratamento } \\
\text { inicial }\end{array}$ & $\begin{array}{l}\text { Conta com um } \\
\text { programa de } \\
\text { extensão da vida útil }\end{array}$ \\
\hline
\end{tabular}

Fonte: Global Health Security Action Group Pandemic Influenza Planning and Preparedness Survey, Canada, 2008 
durante a gripe espanhola (H1N1), entre 1918 e 1920, aproximadamente 40 milhões de pessoas morreram em todo o mundo. A gripe asiática de 1957-1958 matou dois milhões de pessoas. Mais recentemente, entre 1968 e 1969, um milhão de mortes foram causadas pela gripe Hong Kong (H3N2). Se essa é a experiência histórica, a estimativa de mortalidade da OMS para o vírus H5N1 de dois a 7,4 milhões de pessoas pareceu obscura.

A droga Oseltamivir foi desenvolvida pela companhia norte-americana Gilead Sciences, ${ }^{(11)}$ que concedeu, com exclusividade, a licença de produção para a suíça Roche em 1996, pelo período de validade da patente, que é de 20 $\operatorname{anos}^{(12)}$. Em 2000, muito antes da ameaça de pandemia, a Roche considerou a possibilidade de retirar o medicamento do mercado, por conta do baixo volume de vendas mundial. Em termos gerais, o Oseltamivir é como uma aspirina mais sofisticada, utilizado para atacar e diminuir os sintomas da gripe.

Em 27 de outubro de 1999, a Food and Drug Administration (FDA) ${ }^{(13)}$ publicou a primeira aprovação para o tratamento das gripes dos tipos A e B sem nenhuma complicação. Em novembro de 2000 , as indicações profiláticas foram estendidas, precisamente um ano após começar a se falar sobre uma possível epidemia e sobre o risco de pandemia da gripe aviária. Agora, como as indicações puderam ser estendidas sem nenhuma oportunidade de realização de ensaios clínicos para comprovar se o medicamento poderia prevenir a infecção e a propagação da doença?

Em 2004, a OMS recomendou a estocagem do Oseltamivir. ${ }^{(14)} \mathrm{Na}$ França, por exemplo, foram armazenados medicamentos para o atendimento de $50 \%$ da população, uma situação nunca vista antes em relação a outras doenças. Vários países em desenvolvimento providenciaram estoques do Oseltamivir, seguindo as orientações da agência internacional, e foram penalizados por isso, pois esses estoques terão que ser destruídos em breve, nos próximos anos. As nações desenvolvidas contam com grandes quantidades de matérias-primas estocadas, que têm validade de 10 a 15 anos, mais tempo do que o produto final, que vale por cinco anos. A maioria dos países em desenvolvimento recebeu o produto final com sua validade de cinco anos, que, em boas condições de armazenagem, pode ser estendida

(11) Um dos principais acionistas dessa companhia farmacêutica, como revelado em vários meios de comunicação na época, era Donald Rumsfeld, que atuou como gerente geral até 2001, quando foi nomeado secretário de Defesa dos Estados Unidos. A imprensa inglesa mencionou que, quando os Estados Unidos decidiram constituir um estoque de Oseltamivir, Rumsfeld teve que abandonar a reunião governamental em razão da possibilidade de conflitos de interesse que a sua presença no encontro poderia representar.

(12) A partir da data de depósito da patente.

(13) Nota do editor: Food and Drug Administration: trata-se da agência governamental dos Estados Unidos, responsável pelo controle e fiscalização de bens e produtos relacionados à saúde. MORAES, Eliana Aparecida da Silva. O poder regulamentar e as competências normativas conferidas à Agência Nacional de Vigilância Sanitária. Revista de Direito Sanitário, São Paulo, v. 2, n. 1, p. 42, mar./jun. 2001. (14) WORLD HEALTH ORGANIZATION. WHO interim guidelines on clinical management of humans infected by influenza A (H5N1). Geneva: WHO, 2004. 
por no máximo mais um ou dois anos. Mas, quando a data de expiração chegar, as drogas terão que ser destruídas.

O Centro para Controle e Prevenção de Doenças de Atlanta, nos Estados Unidos, publicou um estudo sugerindo que "Apesar do grande esforço inicial, os governos devem economizar dinheiro estocando a droga antiviral como um fundo contra uma futura pandemia de gripe". ${ }^{(15)} \mathrm{O}$ estudo, baseado em um modelo matemático, argumentava que, como acontece uma pandemia de gripe a cada 80 anos pelo menos, os governos poderiam economizar mais de 3,50 dólares a cada um dólar investido no medicamento.

Em agosto de 2005, a OMS anunciou o recebimento de três milhões de doses de Oseltamivir da Roche ${ }^{(16)}$, a fim de constituir um estoque. Em seguida, elaborou um manual e um guia com orientações sobre a criação de estoques.

Isso mostra uma interessante estratégia comercial da Roche. Depois de fornecer três milhões de doses para a OMS, recebeu os agradecimentos da diretora geral da instituição, Margareth Chan, que se viu obrigada a iniciar a estocagem do produto. Na manhã seguinte, em um encontro de ministros da Saúde da União Europeia, ratificou-se a importância de se manterem estoques ${ }^{(17)}$, usando como exemplo a própria OMS (referência mundial em assuntos de saúde).

Nesse contexto, uma série de questões emerge. Por que manter um estoque de Oseltamivir em vez de um de drogas antirretrovirais? Por que não preparar o mundo para a AIDS da mesma maneira que para a gripe $A(H 1 N 1)$ ? Por que e como foi anunciada a eficácia de drogas existentes antes de se conhecerem os resultados de testes clínicos em pessoas, uma vez que não houve tempo para desenvolver estes testes? Quando o armazenamento foi iniciado, pouco se sabia sobre a possibilidade de o vírus criar resistência contra o medicamento. Hoje, sabemos que há sérios problemas de desenvolvimento de resistência ${ }^{(18)}$ à droga que indicam que o produto não deve ser maciçamente distribuído(19). Nenhuma referência foi feita por autoridades nacionais ou internacionais sobre os problemas e implicações em caso de uso em grandes proporções.

(15) Jornal publicado pelo Centro para Controle e Prevenção de Doenças dos Estados Unidos. Agosto de 2005.

(16) WORLD HEALTH ORGANIZATION. Donation of three million treatments of oseltamivir to WHO will help early response to an emerging influenza pandemic, cit.

(17) Estoques de antivirais em 30 países europeus que são membros do Sistema de Vigilância da Influenza da Europa (em inglês: European Influenza Surveillance Scheme - EISS). Todos os países, com exceção da Ucrânia, tinham estoques do inibidor da neuraminidase (NAI) Oseltamivir. European Influenza Surveillance Scheme Co-ordination Centre, Netherlands Institute for Health Services Research (NIVEL), Utrecht, Netherlands. a.meijer@nivel.nl. EURO Surveillance: Bulletin Europeen sur les Maladies Transmissibles. European Communicable Disease Bulletin, v. 12, n. 4, p. E3-4, 2007. (18) BAZ, Mariana; ABED, Yacine; PAPENBURG, Jesse; BOUHY, Xavier; HAMELIN, Marie-Ėve; BOIVIN, Guy. Emergence of Oseltamivir-Resistant Pandemic H1N1 Virus during Prophylaxis. The New England Journal of Medicine, n. 361, p. 2296-2297, Dec. 2009.

(19) MOSCANA, Anne. Global Transmission of Oseltamivir-Resistant Influenza. The New England Journal of Medicine, n. 360, p. 953-956, Mar. 2009. 
Além disso, no Japão, houve vários casos de suicídios entre a população jovem de 14 a 25 anos que estava em tratamento com o Oseltamivir. ${ }^{(20)}$

O FDA, a OMS e a Roche fizeram várias observações depois que a estocagem começou. O FDA afirmou claramente que não se considerou nenhum aspecto em relação à preparação para uma pandemia da gripe aviária ou da gripe A. ${ }^{(21)}$

Ao contrário, a OMS disse que "evidências sugerem que algumas drogas antivirais, notadamente o Oseltamivir, podem reduzir a duração da replicação do vírus e melhorar as perspectivas de sobrevivência"(22). Vários documentos da OMS referiam-se ao Oseltamivir como o único medicamento efetivo contra a possibilidade de uma pandemia da gripe aviária. Em relação a este último ponto, vale esclarecer que é questionável se o Oseltamivir era ou não a única droga efetiva; tratou-se de um pronunciamento apressado da OMS, uma vez que a transmissão entre humanos ainda não havia começado. Era conhecida alguma eficácia do medicamento em casos de gripes sazonais, mas, para esse novo tipo de gripe, embora houvesse alguma sensibilidade em exames in vitro, testes clínicos ainda não haviam sido realizados. Mesmo assim, a página na internet da Roche afirma que o remédio é "um dos medicamentos mais importantes disponíveis atualmente para combater a gripe sazonal e a pandêmica". (23)

\section{A SEVERIDADE NÃO FOI CONSIDERADA}

O número estimado de mortes anuais em decorrência da gripe comum sazonal é de 500 mil casos. ${ }^{(24)}$ A OMS reportou 8.449 óbitos ${ }^{(25)}$ causados pela

(20) "Em março de 2007, autoridades japonesas se pronunciaram contra a prescrição do Oseltamivir para adolescentes entre 10 e 19 anos. Essa medida incomum e severa foi tomada em decorrência de dois casos de suicídios de jovens de 14 anos que tomavam o medicamento; 52 outras mortes (14 de crianças e adolescentes) foram associadas à mesma droga. Até agora, a Europa não adotou medidas similares. Quando uma autoridade regulatória instrui os profissionais médicos a não prescreverem um determinado medicamento, mas, ao mesmo tempo, decide não retirar do mercado o produto, médicos e pacientes ficam um pouco confusos." MAXWELL, Simon R. J. Tamiflu and neuropsychiatric disturbance in adolescents. BMJ 2007; 334:1232. doi: 10.1136/bmj.39240.497025.80. June 14th 2007. Disponível em: <http://www.bmj.com/content/334/7606/1232>. Acesso em 29 nov. 2012.

(21) UNITED STATES. Food and Drug Administration. Disponível em: <http://www.fda.gov/Drugs/ DrugSafety/PostmarketDrugSafetyInformationforPatientsandProviders/ucm107838.htm>. Acesso em: 31 jul. 2012.

(22) WORLD HEALTH ORGANIZATION. Avian Influenza. Fact sheet. Apr. 2011. Disponível em: <http://www.who.int/mediacentre/factsheets/avian_influenza/en/index.html>. Acesso em: 31 jul. 2012. (23) ROCHE. Media release. Basel, April 26th, 2007. Disponível em: <http://www.roche.com/medcor-2007-04-26>. Acesso em: 31 jul. 2012.

(24) LA VANGUARDIA. Una nueva ola de gripe A podría llegar a finales de invierno o en primavera, según la OMS. Barcelona, 17 dez. 2009. Disponível em: <http://www.lavanguardia.com/ vida/20091217/53847502323/una-nueva-ola-de-gripe-a-podria-llegar-a-finales-de-invierno-o-enprimavera-segun-la-oms.html>. Acesso em: 4 set. 2012.

(25) WORLD HEALTH ORGANIZATION. Pandemic (H1N1) 2009 - update 112. Weekly update. Geneva, Aug. 6th, 2010. Disponível em: <http://www.who.int/csr/don/2010_08_06/en/index.html>. Acesso em: 31 jul. 2012. 
gripe $A(H 1 N 1)$. Como anunciado pela imprensa e pela literatura científica, a preocupação mais séria foi que a severidade e a mortalidade da doença não foram consideradas na decisão de anunciar a fase 6 da pandemia. ${ }^{(26)}$

Um novo estudo publicado pela revista Lancet(27) estimou que o número de mortes da pandemia de $\mathrm{H} 1 \mathrm{~N} 1$ foi 15 vezes maior do que o inicialmente relatado pela OMS. Enquanto a instituição confirmou o total de 18.449 mortes causadas pelo vírus da gripe, em 2009, os autores da pesquisa sugerem que até 500 mil pessoas poderiam ter morrido. Depois de explicar o modelo estatístico utilizado para contabilizar os óbitos nos países em desenvolvimento, o artigo conclui que será necessário melhorar a resposta global a futuras pandemias e desenvolver a capacidade de produzir vacinas para a gripe em quantidade suficiente para atender o continente africano e o Sudeste asiático. Em alguns países do Hemisfério Norte, mais de $90 \%$ das vacinas disponíveis não foram utilizadas e tiveram que ser destruídas, como discutiremos a seguir. Diz-se que os estatísticos são indivíduos que "torturam" dados até que eles confessem a verdade.

Em setembro de 2010, a diretora geral da OMS anunciou o fim da pandemia e, assim, a publicação de estatísticas também foi finalizada. Agora, por que parar a divulgação de estatísticas, quando seria muito melhor manter o monitoramento sobre o vírus para poder afirmar que a pandemia realmente chegou ao fim?

Em relação à comunicação de estatísticas e casos, havia uma situação que, de alguma maneira, parecia indecente e embaraçosa. A OMS montou uma espécie de sala de operações em Genebra, com pessoas trabalhando em turnos que permitiam o monitoramento de casos de mortes e de riscos da gripe 24 horas por dia. Eram feitas transmissões ao vivo, na base de caso a caso, em todo o mundo. Qual era a justificativa para essa comunicação rápida? Qual era o problema em esperar 24 ou 48 horas para divulgar a informação, sabendo que a taxa de mortalidade estava muito baixa desde o início? Mortes provocadas por doenças com grande potencial de óbito são usualmente reportadas pela OMS uma vez por ano.

(26) WALSH, Bryan. The H1N1 Flu: is this a Pandemic, or Isn't It? Time Health, June 10th, 2009. Disponível em: <http://www.time.com/time/health/article/0,8599,1903712,00.html\#ixzz1aqtkyNLl>. Acesso em: 31 jul. 2012.

(27) DAWOOD, Fatimah S. et al. Estimated global mortality associated with the first 12 months of 2009 pandemic influenza A H1N1 virus circulation: a modeling study. The Lancet Infectious Disease, v. 12, n. 9, p. 687-695, Sept. 2012. Disponível em: <http://www.thelancet.com/journals/laninf/article/ PIIS1473-3099(12)70121-4/fulltext>. Acesso em: 31 jul. 2012. 


\section{COMO PAÍSES EUROPEUS CALCULARAM O NÚMERO NECESSÁRIO DE VACINAS}

Seguindo a mesma lógica do gerenciamento da pandemia, vamos analisar como países europeus (altamente sofisticados, com capacidade para fazer todo o planejamento necessário) calcularam a quantidade de vacinas necessárias para enfrentar a situação.

Tabela 2 - Compras governamentais de vacinas para H1N1

\begin{tabular}{|l|c|c|}
\hline Países & \multicolumn{1}{c}{$\begin{array}{c}\text { População } \\
\text { (milhão) }\end{array}$} & $\begin{array}{c}\text { Número de vacinas } \\
\text { compradas (milhão) }\end{array}$ \\
\hline ALEMANHA & 82 & 50 \\
\hline BÉLGICA & 10 & 12,6 \\
\hline ESPANHA & 47 & 37 \\
\hline FRANÇA & 60 & 94 \\
\hline ITÁLIA & 60 & 48 \\
\hline HOLANDA & 16 & 34 \\
\hline SUÍÇA & 7 & 13 \\
\hline
\end{tabular}

Diversas fontes

A Alemanha, com 82 milhões de habitantes, comprou 50 milhões de vacinas. Por quê? A Bélgica, com 10 milhões, adquiriu 12,6 milhões de doses. A França é um caso muito interessante, pois, com 60 milhões de cidadãos, comprou 94 milhões de doses. Já Suíça e Holanda compraram o dobro de doses em relação ao tamanho de suas populações. ${ }^{(28)}$

No caso francês, o parlamento realizou uma investigação sobre a condução da pandemia. O inquérito questionou o ministro da Saúde sobre a compra de 94 milhões de doses de vacina ${ }^{(29)}$ e a justificativa dada foi a de que a população poderia precisar de uma segunda dose. Ainda assim, a quantidade comprada parece arbitrária - mais do que o suficiente para oferecer a todo cidadão uma dose única, porém, não o bastante para vacinar, pela segunda vez, cada um dos habitantes do país. Quando a pandemia acabou, a informação oficial do

(28) VELASQUEZ, Germán. Cuánto costó la "vacuna contra la H1N1"? Mémoire des luttes, 26 Oct. 2010. Disponível em: <http://www.medelu.org/Cuanto-costo-la-vacuna-contra-la>. Acesso em: 31 jul. 2012.

(29) LA FRANCE achète 94 millions de vaccins contre la grippe A. L'Express, Paris, 16 July 2009. Disponível em: <http://www.lexpress.fr/actualite/sciences/sante/la-france-achete-94-millions-devaccins-contre-la-grippe-a_774833.html>. Acesso em: 31 jul. 2012 
Ministério da Saúde francês indicava que somente seis milhões de pessoas haviam sido imunizadas.

No início, anunciou-se que as doses excedentes dos países desenvolvidos seriam doadas para as nações mais pobres, mas alguns países no Hemisfério Sul não demonstraram nenhuma intenção de receber esse presente, uma vez que a gripe não se mostrava um risco para eles, em razão das altas temperaturas em seus territórios (de $30^{\circ} \mathrm{C}$ a $35^{\circ} \mathrm{C}$ na maioria dos países africanos), o que diminui a possibilidade de desenvolvimento da gripe em comparação com as nações do Hemisfério Norte.

Em relação ao Oseltamivir, como dito, não foram realizados testes clínicos em humanos que justificassem a profilaxia em caso de uma pandemia da gripe A ( $\mathrm{H} 1 \mathrm{~N} 1)$. Os cientistas informaram que o vírus tinha uma grande capacidade de mutação e sugeriram que as cepas mutantes poderiam ser piores. Porém, eles não mencionaram que essa gripe poderia se autoextinguir, o que aconteceu com outras formas de gripes aviárias, mais notadamente com o vírus $\mathrm{H} 7 \mathrm{~N} 7 \mathrm{da}$ gripe holandesa em $2003^{(30)}$. No fim, também foi esse o caso da gripe A, que aparentemente diminuiu sua intensidade e praticamente desapareceu.

Nos Estados Unidos registrou-se um enorme desperdício, com a incineração de 40 milhões de doses de vacinas contra o vírus $\mathrm{H} 1 \mathrm{~N} 1$, no valor estimado de 260 milhões de dólares ${ }^{(31)}$. Somente $6 \%$ das vacinas compradas pelo governo francês para o H1N1 foram usadas. As doses restantes terão que ser destruídas.

É difícil entender quais as razões de saúde pública para justificar compras maciças de vacinas contra a gripe A pelos países do Hemisfério Norte.

Como reportado por Deborah Cohen e Philip Carter ${ }^{(32)}$, alguns especialistas advertiram a OMS de que o gerenciamento da pandemia teve ligações claras com as empresas farmacêuticas produtoras de antivirais e vacinas. De acordo com Cohen e Carter, o guia da OMS sobre o uso de antivirais em uma pandemia foi autorizado por um especialista em gripe que, ao mesmo tempo, estava recebendo pagamentos da Roche, produtora do Oseltamivir (Tamiflu), pelo trabalho de consultoria e palestras. ${ }^{(33)}$

(30) H7N7 Dutch Avian Flu Outbreak (2003) Associated with Human Conjunctivitis and Fatal Case of Acute Respiratory Distress Syndrome in Exposed Veterinarian Who Did Not Take Tamiflu or Receive Vaccination. Bio Report, Illinois, n. 345, 2 Apr. 2006.

(31) "Milhões de doses da vacina contra o vírus H1N1 expiraram na quarta-feira e tiveram que ser destruídas, segundo o Departamento de Saúde e de Serviços Humanos dos Estados Unidos. Milhões mais ainda estam armazenadas e irão vencer em diferentes momentos no próximo ano." DUDA, Kristina. Millions of Doses of H1N1 Vaccine Destroyed. About.com. 2 July 2010. Disponível em: $<$ http://coldflu.about.com/b/2010/07/02/millions-of-doses-of-h1n1-vaccine-destroyed.htm>. Acesso em: 31 jul. 2012.

(32) COHEN, Deborah; CARTER, Philip. Conflicts of interest. WHO and the pandemic flu "conspiracies". British Medical Journal, 3 June 2010. Disponível em: <http://www.ncbi.nlm.nih.gov/ pubmed/20525679>. Acesso em: 31 jul. 2012.

(33) Id. Ibid. 


\section{CONSIDERAÇÕES FINAIS}

Houve confusão e uma combinação complexa de interesses de diferentes, e algumas vezes opostos, atores. O primeiro grupo: a OMS e as autoridades sanitárias nacionais que legitimamente tinham o objetivo de proteger a população, a partir de valores e perspectivas da saúde pública, mesmo considerando os erros cometidos, como, por exemplo, o fato de que não se informou a população de que lavar as mãos com soluções alcoólicas era inútil e que o melhor era usar água e sabão para higienizá-las. Milhões de dólares foram gastos na compra dessas substâncias.

O segundo grupo: os governos e os líderes políticos que, talvez, não tivessem como prioridade as questões de saúde pública e estivessem mais interessados em proteger ou promover a si mesmos, a partir de um ponto de vista político. Na Europa, por exemplo, ainda existe o fantasma do caso do sangue contaminado, quando alguns gestores públicos altamente credenciados foram responsabilizados judicialmente.

O terceiro grupo: a indústria farmacêutica, obviamente, guiada por interesses comerciais.

De acordo com o relatório do Conselho Europeu, "O tratamento da pandemia do H1N1: necessidade de mais transparência", as relações e interações entre esses três grupos não foram sempre claras nos níveis nacional e internacional, o que trouxe complicações para o gerenciamento da pandemia da gripe $\mathrm{A}(\mathrm{H} 1 \mathrm{~N} 1) .{ }^{\left({ }^{(34)}\right.}$

Concluindo, houve respostas positivas e negativas para a pandemia. Do lado positivo, deu-se um processo de educação sanitária mundial sem precedentes. Em poucos dias/horas, autoridades sanitárias e a imprensa enviaram uma mensagem clara: havia um perigo e havia risco. Isso funcionou perfeitamente. Também ficou demonstrado que, em caso de um risco global, existem recursos para serem usados, como pôde ser visto com as grandes quantias de dinheiro gastas e com a criação de um mercado de imunização de 400 bilhões de dólares ${ }^{(35)}$, sem considerar o álcool utilizado em soluções, as máscaras e alternativas mais baratas.

(34) FLYNN, Paulo. The handling of the H1N1 pandemic: more transparency needed. Report Social Health and Family Affairs Committee, United Kingdom: Counsel of Europe, 23 Mar. 2010. Disponível em: <http://assembly.coe.int/CommitteeDocs/2010/20100329_MemorandumPandemie_E.pdf>. Acesso em: 31 jul. 2012.

(35) "'Os produtores de vacinas poderiam fabricar 4,9 bilhões de doses para a pandemia de gripe por ano, no melhor cenário', afirmou Margaret Chan, diretora geral da OMS, segundo a Reuters, em 21 de julho de 2009. Países mais saudáveis como os Estados Unidos e a Bretanha pagarão menos do que 10 dólares por dose (da vacina para o H1N1)... Países em desenvolvimento pagarão um preço mais baixo (cerca de 400 bilhões de dólares para a indústria farmacêutica), segundo a Business Week, de julho de 2009". CHOSSUDOVSKY, Michel. The Worldwide H1N1 Swine Flu Pandemic..., 4 Aug. 2009. Global Research. NWO Observer. Disponível em: <http://nwoobserver.wordpress. com/2009/08/04/the-worldwide-h1n1-swine-flu-pandemic/>. Acesso em: 31 jul. 2012. 
Do lado negativo, o conceito de imunização foi colocado em risco. A mais importante ferramenta terapêutica da saúde pública é a capacidade de prevenir uma doença, e nós não sabemos ainda quais as consequências dessa falha. Mensagens foram inapropriadamente divulgadas e pôde-se constatar um problema de comunicação entre a mídia e a comunidade científica. Por exemplo, na França e na Espanha os trabalhadores da Saúde se recusaram a tomar a vacina ${ }^{(36)} \mathrm{e}$ isso foi estampado nas primeiras páginas de muitos jornais. Os funcionários de um hospital disseram claramente "nós não iremos tomar a vacina"(37), uma afirmação que pode influenciar de maneira prejudicial a opinião pública quanto à vacinação e ações relacionadas à saúde pública.

Finalmente, a OMS, os ministros da Saúde de diversos países e algumas comunidades científicas se comprometeram devido a decisões falhas em face de uma crise de saúde pública(38), resultando em uma perda significativa de credibilidade. Não se sabe ainda como as autoridades da OMS e a dos ministérios foram afetadas e como essa perda de credibilidade vai influenciar a habilidade em conduzir problemas semelhantes no futuro.

Países do Hemisfério Norte gastaram bilhões de dólares com a pandemia. E, no fim, os perdedores nessa situação foram os pacientes das nações em desenvolvimento, que estão morrendo todos os dias em razão da falta de acesso a medicamentos dos quais que eles necessitam.

\section{REFERÊNCIAS}

ASSEMBLÉE NATIONALE. Rapport. Au nom de la Commission d'enquête sur la manière dont a été programmée, expliquée et gérée la campagne de vaccination contre la grippe $A(H 1 N 1)$. Disponível em: <http://www.assemblee-nationale.fr/13/ rap-enq/r2698.asp>. Acesso em: 26 jul. 2012.

BAZ, Mariana; ABED, Yacine; PAPENBURG, Jesse; BOUHY, Xavier; HAMELIN, Marie-Ėve; BOIVIN, Guy. Emergence of Oseltamivir-Resistant Pandemic H1N1 Virus during Prophylaxis. The New England Journal of Medicine, n. 361, p. 2296-2297, Dec. 2009.

(36) CANNET, Didier. Le vaccin contre la grippe H1N1 suscite méfiance et toujours autant de questions. Lemonde.Fr, 20 Nov. 2009. Disponível em: <http://www.lemonde.fr/planete/article_ interactif/2009/11/20/le-vaccin-contre-le-grippe-h1n1-suscite-mefiance-et-toujours-autant-dequestions_1269695_3244.html>. Acesso em: 31 jul. 2012.

(37) WYDERKO, Kasia. Francia rechaza la vacuna contra influenza AH1N1. Noticieros Televisa, 12 nov. 2009.

(38) FLYNN, Paulo. op. cit. 
CANNET, Didier. Le vaccin contre la grippe H1N1 suscite méfiance et toujours autant de questions. Lemonde.Fr, 20 Nov. 2009. Disponível em: <http://www. lemonde.fr/planete/article_interactif/2009/11/20/le-vaccin-contre-le-grippe-h1n1suscite-mefiance-et-toujours-autant-de-questions_1269695_3244.html>. Acesso em: 31 jul. 2012.

CASSEL, Ingri. H1N1 pandemic scam cost \$billions worldwide. European Parliamentary Assembly holds public hearing to investigate "what went wrong". Idaho Observer, Strasbourg, France. Feb. 10th, 2010. Disponível em: <http:// www.proliberty.com/observer/20100234.html>. Acesso em: 26 jul. 2012.

CENTER FOR DISEASE CONTROL AND PREVENTION. Transmission of Influenza A Viruses Between Animals and People. Subtypes that have caused widespread illness in people either in the past or currently are H3N2, H2N2, H1N1, and H1N2. Disponível em: <http://www.cdc.gov/flu/avian/gen-info/transmission. $\mathrm{htm}>$. Acesso em: 11 nov. 2012.

CHOSSUDOVSKY, Michel. The Worldwide H1N1 Swine Flu Pandemic..., 4 Aug. 2009. Global Research. NWO Observer. Disponível em: <http://nwoobserver. wordpress.com/2009/08/04/the-worldwide-h1n1-swine-flu-pandemic/>. Acesso em: 31 jul. 2012.

COHEN, Deborah; CARTER, Philip. Conflicts of interest. WHO and the pandemic flu "conspiracies". British Medical Journal, 3 June 2010. Disponível em: <http:// www.ncbi.nlm.nih.gov/pubmed/20525679>. Acesso em: 31 jul. 2012.

DAWOOD, Fatimah S. et al. Estimated global mortality associated with the first 12 months of 2009 pandemic influenza A H1N1 virus circulation: a modeling study. The Lancet Infectious Disease, v. 12, n. 9, p. 687-695, Sept. 2012. Disponível em: <http://www.thelancet.com/journals/laninf/article/PIIS1473-3099(12)70121-4/ fulltext>. Acesso em: 31 jul. 2012.

DUDA, Kristina. Millions of Doses of H1N1 Vaccine Destroyed. About.com. 2 July 2010. Disponível em: <http://coldflu.about.com/b/2010/07/02/millions-of-dosesof-h1n1-vaccine-destroyed.htm>. Acesso em: 31 jul. 2012.

EURO Surveillance: Bulletin Europeen sur les Maladies Transmissibles. European Communicable Disease Bulletin, v. 12, n. 4, p. E3-4, 2007.

FLYNN, Paulo. The handling of the H1N1 pandemic: more transparency needed. Report Social Health and Family Affairs Committee, United Kingdom: Counsel of Europe, 23 Mar. 2010. Disponível em: <http://assembly.coe.int/ CommitteeDocs/2010/20100329_MemorandumPandemie_E.pdf >. Acesso em: 31 jul. 2012.

H7N7 Dutch Avian Flu Outbreak (2003) Associated with Human Conjunctivitis and Fatal Case of Acute Respiratory Distress Syndrome in Exposed Veterinarian Who Did Not Take Tamiflu or Receive Vaccination. Bio Report, Illinois, n. 345, 2 Apr. 2006. 
LA FRANCE achète 94 millions de vaccins contre la grippe A. L'Express, Paris, 16 July 2009. Disponível em: <http://www.lexpress.fr/actualite/sciences/sante/lafrance-achete-94-millions-de-vaccins-contre-la-grippe-a_774833.html>. Acesso em: 31 jul. 2012.

LA VANGUARDIA. Una nueva ola de gripe A podría llegar a finales de invierno o en primavera, según la OMS. Barcelona, 17 dez. 2009. Disponível em: <http:// www.lavanguardia.com/vida/20091217/53847502323/una-nueva-ola-de-gripe-apodria-llegar-a-finales-de-invierno-o-en-primavera-segun-la-oms.html>. Acesso em: 4 set. 2012 .

MAXWELL, Simon R. J. Tamiflu and neuropsychiatric disturbance in adolescents. BMJ 2007; 334:1232. doi: 10.1136/bmj.39240.497025.80. June 14th 2007. Disponível em: <http://www.bmj.com/content/334/7606/1232>. Acesso em 29 nov. 2012.

MORAES, Eliana Aparecida da Silva. O poder regulamentar e as competências normativas conferidas à Agência Nacional de Vigilância Sanitária. Revista de Direito Sanitário, São Paulo, v. 2, n. 1, p. 39-56, mar./jun. 2001.

MOSCANA, Anne. Global Transmission of Oseltamivir-Resistant Influenza. The New England Journal of Medicine, n. 360, p. 953-956, Mar. 2009.

ROCHE. Media release. Basel, April 26th, 2007. Disponível em: <http://www. roche.com/med-cor-2007-04-26>. Acesso em: 31 jul. 2012.

UNAIDS. Global Report 2010. Disponível em: <http://www.unaids.org/ globalreport/Global_report.htm>. Acesso em: 26 jul. 2012.

UNITEDSTATES. Food and DrugAdministration Disponívelem:<http://www.fda.gov/ Drugs/DrugSafety/PostmarketDrugSafetylnformationforPatientsandProviders/ ucm107838.htm>. Acesso em: 31 jul. 2012.

VELASQUEZ, Germán. Cuánto costó la "vacuna contra la H1N1"? Mémoire des luttes, 26 Oct. 2010. Disponível em: <http://www.medelu.org/Cuanto-costo-lavacuna-contra-la>. Acesso em: 31 jul. 2012.

WALSH, Bryan. The H1N1 Flu: is this a Pandemic, or Isn't It? Time Health, June 10th, 2009. Disponível em: <http://www.time.com/time/health/ article/0,8599,1903712,00.html\#ixzz1aqtkyNLl>. Acesso em: 31 jul. 2012.

WORLD HEALTH ORGANIZATION. Avian Influenza. Fact sheet. Apr. 2011. Disponível em: <http://www.who.int/mediacentre/factsheets/avian_influenza/en/ index.html>. Acesso em: 31 jul. 2012.

Current WHO phase of pandemic alert for Pandemic (H1N1) 2009. Disponível em: <http://www.who.int/csr/disease/swineflu/phase/en>. Acesso em: 4 set. 2012.

Donation of three million treatments of oseltamivir to WHO will help early response to an emerging influenza pandemic. Aug. 24th 2005. Disponível em: <http:// www.who.int/mediacentre/news/releases/2005/pr36/en/>. Acesso em: 27 jul. 2012. 
Medicines: medicines for children. Fact Sheet n. 341, June 2010. Disponível em: <http://www.who.int/mediacentre/factsheets/fs341/en/index. html>. Acesso em: 26 jul. 2012.

Pandemic (H1N1) 2009 - update 112. Weekly update. Geneva, Aug. 6th, 2010. Disponível em: <http://www.who.int/csr/don/2010_08_06/en/index. html>. Acesso em: 31 jul. 2012.

WHO interim guidelines on clinical management of humans infected by influenza A (H5N1). Geneva: WHO, 2004.

WORLD HEALTH ORGANIZATION/GIP. Cumulative number of confirmed human cases of avian influenza H5N1 reported to WHO, 2003-2011. Aug. 19th 2011. Disponível em: <http://www.who.int/influenza/human_animal_interface/EN_Gl P_20110819CumulativeNumberH5N1casesN.pdf>. Acesso em: 27 jul. 2012.

WYDERKO, Kasia. Francia rechaza la vacuna contra influenza AH1N1. Noticieros Televisa, 12 nov. 2009. 